\title{
Disseminated Tuberculosis Following Total Knee Arthroplasty in an HIV Patient
}

\author{
J. Marschall, J.-M. Evison, S. Droz, U.C. Studer, S. Zimmerli
}

\begin{abstract}
Skeletal tuberculosis is now uncommon in developed countries. In immunocompromised patients - particularly in the HIV-infected - who present with subacute or chronic joint pain refractory to conventional treatment, osteoarticular tuberculosis should still be included in the differential diagnosis. We report on a lethal case of disseminated tuberculosis in an HIV-infected subject. Dissemination may have resulted from the implantation of an articular prosthesis in a knee joint with unsuspected osteoarticular tuberculosis. The diagnosis was established months later when the patient presented with far-advanced tuberculous meningitis, miliary tuberculosis of the lungs, femoral osteomyelitis and extended cold abscesses along the femoral shaft. Failure to respond to a conventional four-drug regimen is explained by the resistance pattern of his multi-drug resistant strain of Mycobacterium tuberculosis, which was only reported after the patient's death. This case illustrates the diagnostic challenges of osteoarticular tuberculosis and the consequences of a diagnostic delay in an HIV-infected individual.
\end{abstract}

Infection 2008; 36: 274-278

DOI 10.1007/s15010-007-7011-1

\section{Introduction}

Skeletal tuberculosis is uncommon in developed countries. Because its incidence has increased with the recent re-emergence of tuberculosis along with the HIV/AIDS pandemic, physicians are increasingly likely to be confronted with this disease and the considerable diagnostic problems it may pose.

HIV infection is the single most important risk factor for the reactivation of latent tuberculosis or the progression of active disease [1]. Thus, tuberculosis - including extrapulmonary forms, which are more common in the HIV-infected - must be included in the differential diagnosis of a wide spectrum of disease manifestations in patients with advanced HIV infection [2-4].

Globally, $1-3 \%$ of patients with active tuberculosis are thought to have osteoarticular manifestations [5].
Indeed, bone and/or joint were the third most common site of extrapulmonary tuberculosis (after pleura and lymph nodes) in the latest survey in the U.S. [6]. Manifestations of skeletal tuberculosis may closely resemble other diseases. A high index of suspicion is therefore needed to establish the correct diagnosis. In immunocompromised patients - particularly in the HIV-infected who present with subacute or chronic articular pain refractory to conventional treatment, osteoarticular tuberculosis should be included in the differential diagnosis.

\section{Case Description}

A 48-year-old HIV-positive man was admitted to the hospital because of fever and confusion that had started a few days earlier. There were no focal neurological deficits or neck stiffness. $\mathrm{He}$ was tachypneic, but auscultation of the lungs was unremarkable and arterial blood gases were normal. Inspection revealed a non-tender swelling of the left knee. The white blood cell count was $9.3 \mathrm{G} / 1$ and his CD4+ cell count was 7 per microliter. The chest X-ray showed micronodular infiltrates suggestive of miliary tuberculosis (Figure 1). A lumbar puncture revealed pleocytosis, low glucose, and elevated protein and lactate levels. An MRI scan of the head showed vasculitis of the basal cerebral arteries and several ischemic brain lesions (Figure 2). Antituberculous treatment with a four-drug regimen was started on the day of admission.

\footnotetext{
J. Marschall, J.-M. Evison

Division for Infectious Diseases, University Hospital Inselspital, Bern, Switzerland

S. Droz, S. Zimmerli (corresponding author)

Institute for Infectious Diseases, University of Bern, Friedbuehlstr. 51, P.O. Box 61, CH-3010 Bern, Switzerland; Phone: (+41/31) 632-3258, Fax: -8766, e-mail: stefan.zimmerli@ifik.unibe.ch

U.C. Studer

Institute for Pathology, University of Bern, Bern, Switzerland

J. Marschall

Division of Infectious Diseases, Washington University School

of Medicine, St. Louis, MO, USA

U.C. Studer

Division of Internal Medicine, Regional Hospital Emmental, Burgdorf, Switzerland
}

Received: December 27, 2006 - Revision accepted: April 17, 2007

Published online: December 14, 2007 


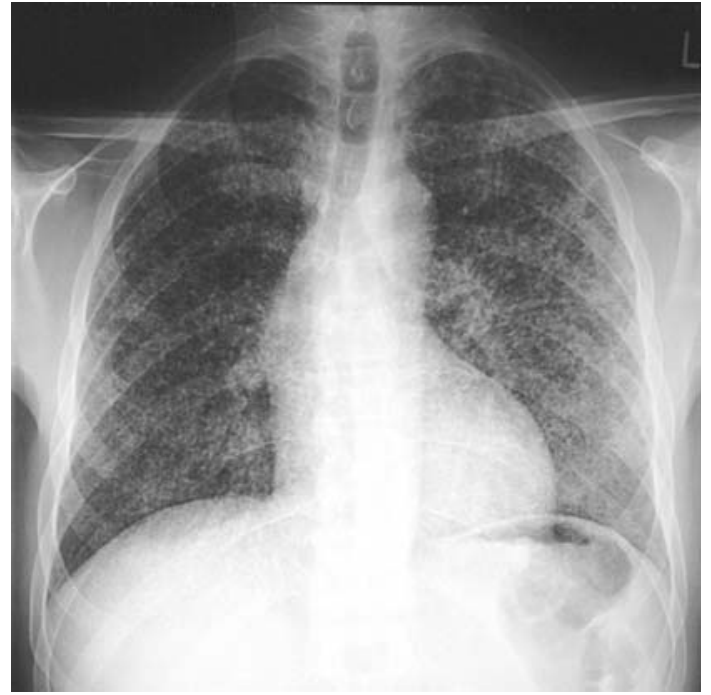

Figure 1. Chest radiograph taken on admission to the hospital: Countless $0.5-1.0 \mathrm{~mm}$ nodules in both lungs typical of miliary tuberculosis.

The patient had been known to be HIV-seropositive since 1987. Under antiretroviral combination therapy, his viral load had been undetectable for years. His CD4+ cell count, however, failed to increase above 100 per microliter and he had been on trimethoprim/sulfamethoxazole (TMP/SMX) prophylaxis since 1988. In 2001, after a minor trauma, he began to suffer from recurrent effusions of the left knee that were thought to be due to meniscal lesions. Two arthroscopies with partial meniscectomy were performed in 2001 and 2002 without success. No microbiological or histopathological examinations were done at that time. A subsequent radiograph showed narrowing of the joint space and a cortical erosion along the lateral femur condyle, suggestive of chronic osteoarthritis (Figure 3). In June 2003, 9 months before admission, total left knee arthroplasty was performed.

Three months before admission, he presented to the outpatient clinic with a painless swelling of the left knee. There was no joint effusion, but an indurated soft-tissue mass measuring $5 \times 10 \mathrm{~cm}$ extended proximally from the knee along the medial aspect of the thigh. The range of motion of the knee joint was reduced. CRP was $54 \mathrm{mg} / \mathrm{l}$. Aspiration of the mass did not grow any bacteria during 5 days of cultivation (under TMP/SMX). Cultures for mycobacteria were not done. A conventional radiograph showed an enlarging cortical erosion of the femur next to the knee prosthesis (Figure 3). An MRI scan of the knee also revealed a polycyclic mass spreading along the femur and inflammation of the femoral medulla (Figure 4). TMP/SMX was stopped to obtain meaningful bacteriology results from a planned biopsy of the mass. However, biopsy could not be performed until the day the patient presented to the emergency room.

All samples taken at the time of admission (of the mass in the left thigh, the patella, joint fluid from the left knee, as well as respiratory secretions, lung biopsy, and CSF) were positive for Mycobacterium tuberculosis by smear microscopy, by the amplified Mycobacterium tuberculosis direct test (Gen-Probe, San Diego, CA, USA), and by culture. Smears stained with auramin-rhodamine showing acid-fast bacilli were confirmed by Ziehl-Neelsen staining. Lowenstein-Jensen slants containing pyruvate and BacT/ALERT mycobacteria process bottles with mycobacteria antibiotic supplement (Organon Teknika, Durham, NC, USA) were used for culture.

The patient was treated with isoniazid, ethambutol, pyrazinamid, and moxifloxacin. Rifampin was not included because of its interactions with his antiretrovirals. Dexamethasone was added for tuberculous meningitis [7]. Due to the patient's poor condition, surgical revision of the knee was postponed. When there was no clinical response after 2 weeks, the antiretroviral therapy was discontinued and moxifloxacin was replaced by rifampin. Streptomycin, an alternative first-line agent was not given because of its potential for ototoxicity. Three weeks into the antituberculous treatment, the patient became comatose. By CT there were widespread ischemic cerebral lesions. Eventually, he died 1 month after admission. Autopsy revealed inflammation of the base of the brain, purulent meningitis, and ischemia of several brain areas. In both lungs there was a large number of small caseating granulomas, but Langhans giant cells were absent. No primary focus of pulmonary tuberculosis could be identified and necrotic hilar lymphnodes were absent. Antimicrobial resistance testing by the
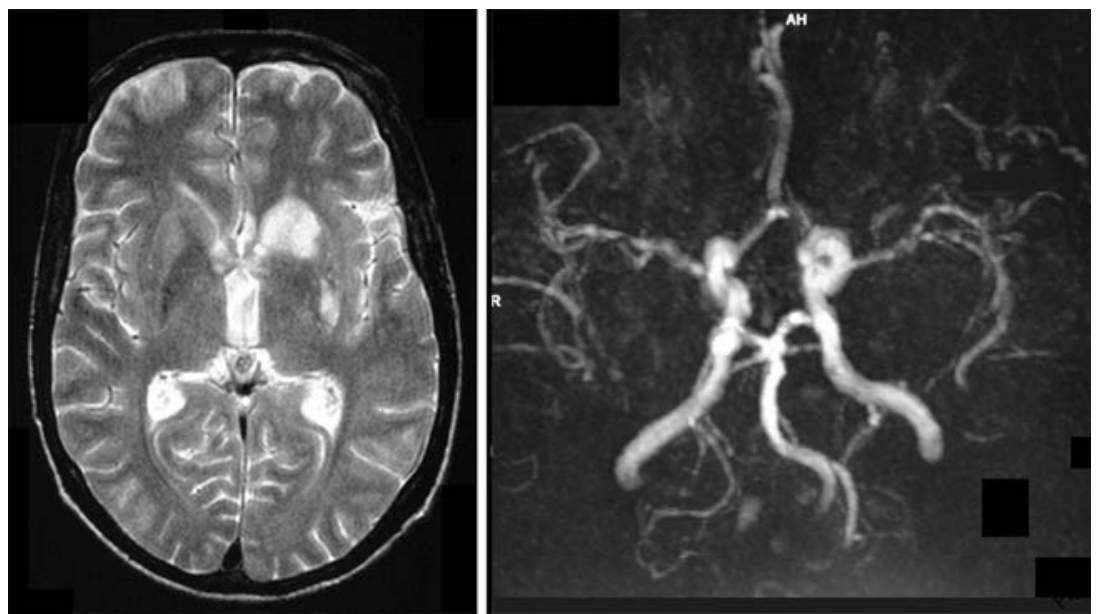

Figure 2. Magnetic resonance imaging study of the head: This T2-weighted image shows hyperintense lesions in the left caput nuclei caudati and in the left putamen compatible with recent ischemic lesions. Time-of-flight angiography demonstrates irregular calibrations ("string of beads") of the medial cerebral arteries, indicating vasculitis. 
radiometric method in a BACTEC 460TB instrument (Beston Dickinson Microbiology Systems, Sparks, MD, USA) revealed that the mycobacteria were resistant to all first-line antituberculous agents and sensitive to ofloxacin.

\section{Discussion}

This case of osteoarticular tuberculosis was complicated by disseminated disease, severe immunodeficiency, and a multi-drug resistant (MDR) organism [8]. The clinical manifestations, the time course, the imaging studies, and the autopsy findings suggest that disseminated infection originated from a reactivated tuberculous focus in the femoral metaphysis that had extended locally into the knee joint. Orthopedic surgery to the knee may have contributed to hematogenous dissemination and local extension along the femoral shaft.

Osteoarticular tuberculosis originates from hematogenous seeding of mycobacteria from the primary pulmonary lesion to where blood supply is best - i.e., the vertebrae, predominantly, and the metaphysis of the long
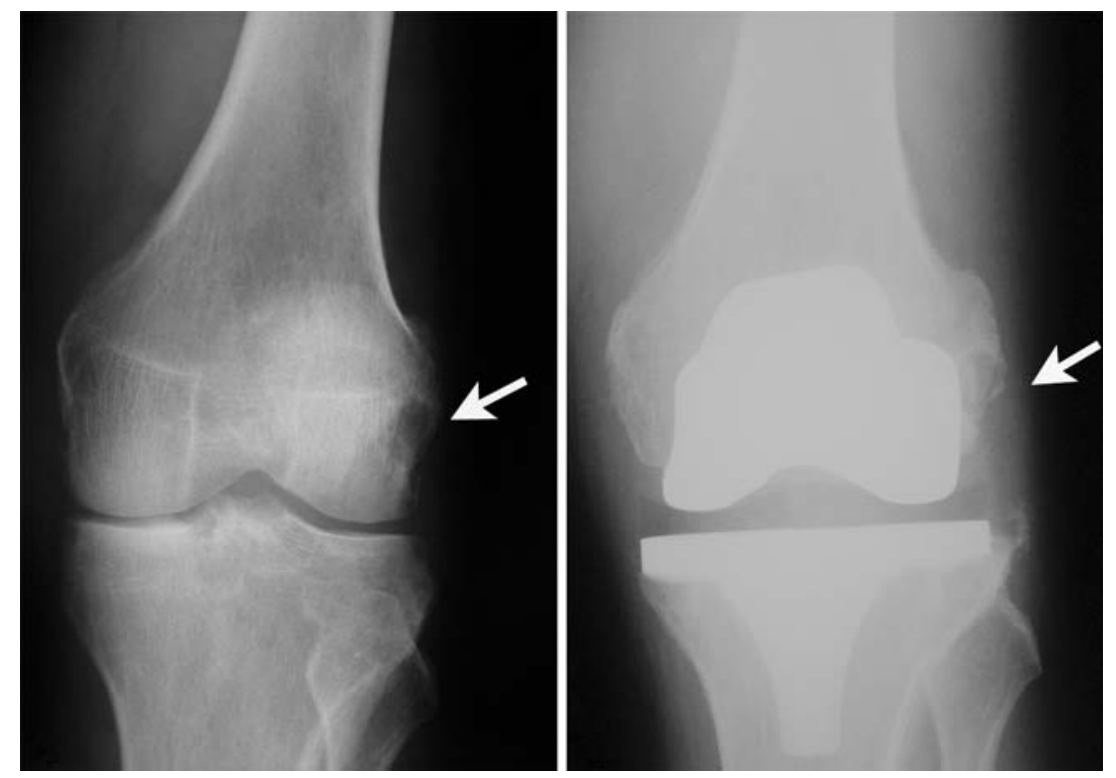

Figure 3. Conventional radiographs of the left knee: The radiograph on the left was taken in January 2003,5 months before total knee arthroplasty. It shows significant narrowing of the joint space and a cortical erosion of the lateral femoral condyle (white arrow) that appears larger on the radiograph on the right that was taken in July 2003,1 month after arthroplasty.
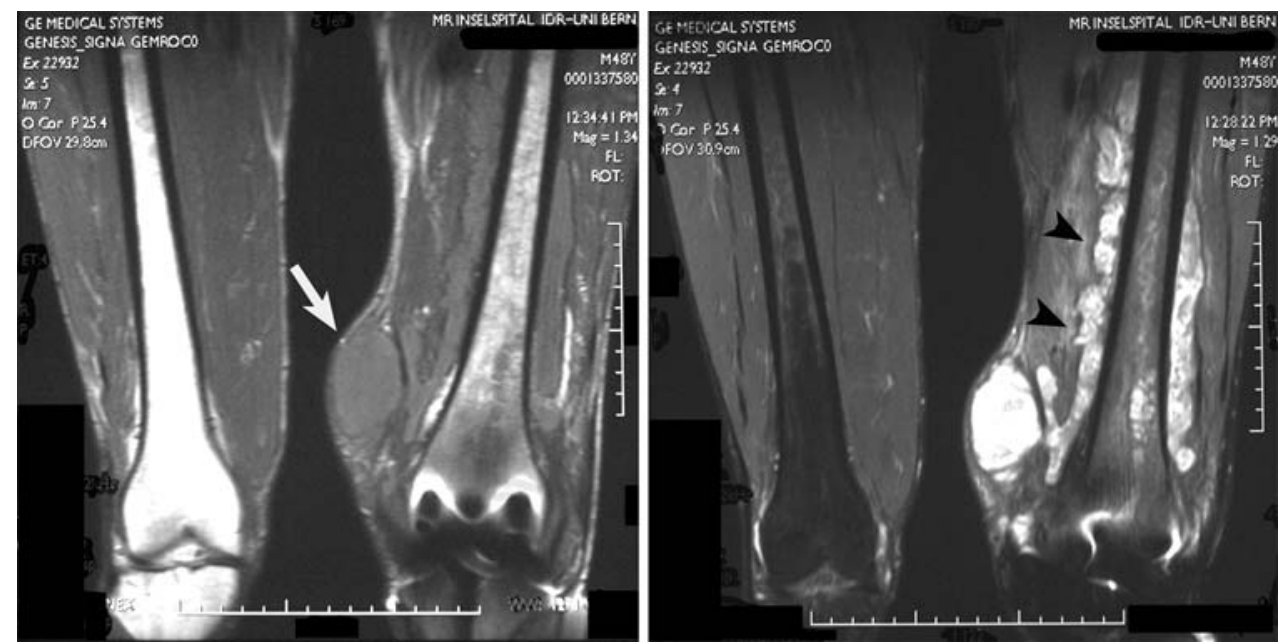

Figure 4. Magnetic resonance imaging study of the left knee: In T1-weighted images a hypointense lesion on the medial side of the distal femur can be seen (white arrow). The same lesion appears hyperintense in T2-weighted sequences and communicates with several other lesions extending along the femur (black arrowheads). The bone marrow's signaling pattern suggests osteomyelitis. Due to artifacts caused by the prosthesis connection of the lesions with the joint space cannot be demonstrated. 
bones. Active disease can develop immediately or arise from latent tuberculosis years later. Joints are rarely involved by direct seeding but rather as a consequence of adjacent tuberculous osteomyelitis [5]. Weight-bearing joints constitute at least $80 \%$ of extraspinal skeletal tuberculosis [9], and the knee is affected in up to $20 \%$ [10]. Monoarticular manifestation is the rule and synovial inflammation and joint effusion are common. The onset of symptoms with joint pain and a limited range of motion is generally insidious. Cartilage and bone destruction caused by synovial granulation tissue preserves the weight-bearing structures in joints considerably, but sclerosis and fibrous ankylosis may ultimately develop [11]. Untreated infection may extend to adjacent soft tissue to form cold abscesses with or without draining sinuses. Both signs are strongly suggestive of tuberculosis, although superinfection of sinuses may obscure the diagnosis [12].

Tuberculosis in a prosthetic joint is usually caused by one of the following mechanisms. Active osteoarticular tuberculosis present at the time of surgery [13, 14], hematogenous seeding from a reactivated focus elsewhere $[15,16]$, or reactivation of latent osteoarticular tuberculosis following arthroplasty $[15,17,18]$. Surgery or traumas are considered to be risk factors for reactivation [19-21]. Prosthetic infection may present months or decades after surgery [15] as implant loosening or low-grade infection $[22,23]$ and must be suspected in unexpected failure of arthroplasty.

Tuberculosis should be considered in any subacute joint or bone pain unresponsive to conventional therapy, particularly in HIV-infected subjects. Osteoarthritis is a common misdiagnosis [18] because its radiological signs resemble Phemister's triad of joint tuberculosis (joint space narrowing, subchondral osteopenia, lateral osseous erosions). Bone lesions can be mistaken for osteomyelitis due to other bacteria or fungi, particularly in the immunocompromised host, and for neoplastic diseases.

Microscopy and culture of tissue samples is the diagnostic gold standard. In cases of tuberculous arthritis, the yield of synovial biopsy exceeds $90 \%$; smears of synovial membrane are positive for acid-fast organisms in 20-40\%, and culture grows mycobacteria in up to $80 \%$ [9]. Smears of joint fluid are less sensitive [24] and the synovial fluid usually shows non-specific signs of inflammation. Histology often reveals granulomas suggestive of, but not specific for, tuberculosis. In HIV infection granulomas may be less well defined [25].

Imaging studies are useful, but no sign is pathognomonic for osteoarticular tuberculosis. The radiological signs include narrowing of the joint space, subchondral erosions, cysts, and osteopenia [26, 27]. Marginal erosions are typical for tuberculosis, as is the absence of early sclerosis. MRI may show inflammatory changes in bones and soft tissue as well as osteonecrosis. The delay in diagnosing skeletal tuberculosis is generally in the range of 12 months from the beginning of symptoms [10], so destruction of bone and cartilage are usually evident by then.

The mainstay of treatment is antituberculous chemotherapy, initially with four drugs including rifampin, isoniazid, pyrazinamid and ethambutol. Recommended treatment duration is 6-9 months for drug-sensitive disease. Surgery is useful for abscess drainage, decompression and - after adequate drug treatment - for reconstructive measures. Successful treatment of tuberculosis in a prosthetic joint often requires removal of the implant material $[18,28]$ but revision arthroplasty may then be done. In general, a combined medical and surgical approach is warranted [17].

\section{References}

1. Horsburgh CR: Priorities for the treatment of latent tuberculosis infection in the United States. N Engl J Med 2004; 350: 2060-2067.

2. Aaron L, Saadoun D, Calatroni I, Launay O, Memain N, Vincent V, Marchal G, Dupont B, Bouchaud O, Valere D, Lortholary O: Tuberculosis in HIV-infected patients: a comprehensive review. Clin Microbiol Infect 2004; 10: 388-398.

3. Shafer RW, Kim DS, Weiss JP, Ouale JM: Extrapulmonary tuberculosis in patients with human immunodeficiency virus infection. Medicine 1991; 70: 384-397.

4. Frieden TR, Sterling TR, Munsiff SS, Watt CJ, Dye C: Tuberculosis. Lancet 2003; 362: 887-899.

5. Tuli SM: General principles of osteoarticular tuberculosis. Clin Orthop Res 2002; 398: 11-19.

6. Centers for Disease Control and Prevention: Reported Tuberculosis in the United States, 2003. Atlanta, GA: U.S. Department of Health and Human Services, CDC, September 2004.

7. Thwaites GE, Nguyen DB, Nguyen HD, Hoang TO, Do TT, Nguyen TC, Nguyen $\mathrm{OH}$, Nguyen TT, Nguyen $\mathrm{NH}$, Nguyen TN, Nguyen NL, Nguyen HD, Vu NT, Cao HH, Tran TH, Pham PM, Nguyen TD, Stepniewska K, White NJ, Tran TH, Farrar JJ: Dexamethasone for the treatment of tuberculous meningitis in adolescents and adults. N Engl J Med 2004; 351: 1741-1751.

8. Fischl MA, Daikos GL, Uttamchandani RB, Poblete RB, Moreno JN, Reyes RR, Boota AM, Thompson LM, Cleary TJ, Oldham SA: Clinical presentation and outcome of patients with HIV infection and tuberculosis caused by multiple-drug-resistant bacilli. Ann Intern Med 1992; 117: 184-190.

9. Malaviya AN, Kotwal PP: Arthritis associated with tuberculosis. Best Pract Res Clin Rheum 2003; 17: 319-343.

10. Martini $M$, Ouahes $M$ : Bone and joint tuberculosis: a review of 652 cases. Orthopedics 1988; 11: 861-866.

11. Davidson PT, Horowitz I: Skeletal tuberculosis. Am J Med 1970; 48: 77-84.

12. McCullough CJ: Tuberculosis as a late complication of total hip replacement. Acta Orthop Scand 1977; 48: 508-510.

13. Su JY, Huang TL, Lin SY: Total knee arthroplasty in tuberculous arthritis. Clin Orthop 1996; 323: 181-187.

14. Yoon TR, Rowe SM, Anwar IB, Chung JY: Active tuberculosis of the hip treated with early total hip replacement - a report of 3 cases. Acta Orthop Scand 2001; 72: 419-421. 
15. Tokumoto JIN, Follansbee SE, Jacobs RA: Prosthetic joint infection due to M. tuberculosis: report of three cases. Clin Infect Dis 1995; 21: 134-136.

16. Ueng WN, Shih $\mathrm{CH}, \mathrm{H}$ seuh S: Pulmonary tuberculosis as a source of infection after total hip arthroplasty: a report of two cases. Int Orthop 1995; 19: 55-59.

17. Berbari EF, Hanssen AD, Duffy MC, Steckelberg JM, Osmon DR: Prosthetic joint infection due to Mycobacterium tuberculosis: a case series and review of the literature. Am J Orthop 1998; 27: 219-227.

18. Marmor M, Parnes N, Dekel S: Tuberculosis infection complicating total knee arthroplasty. J Arthoplasty 2004; 19: 397-400.

19. Hugate R, Pellegrini VD: Reactivation of ancient tuberculous arthritis of the hip following total hip arthroplasty. J Bone Joint Surg 2002; 84: 101-105.

20. Krappel FA, Harland U: Failure of osteosynthesis and prosthetic joint infection due to Mycobacterium tuberculosis following a subtrochanteric fracture: a case report and review of the literature. Arch Orthop Trauma Surg 2000; 120: 470-472.

21. Wray CC, Roy S: Arthroplasty in tuberculosis of the knee. Acta Orthop Scand 1987; 58: 296-298.
22. Bruns J, Luessenhop S, Behrens P: Hematogenous tuberculous infection following revision of a loosened total hip replacement. Langenbeck's Arch Surg 1998; 383: 265-268.

23. Lusk RH, Wienke EC, Milligan TW, Albus AE: Tuberculosis and foreign-body granulomatous reactions involving a total knee prosthesis. Arthr Rheum 1995; 38: 1325-1327.

24. Lange CG, Getty PJ, Morrissey AB, George AL, Young PC, Armitage KB: Destructive osteoarthritis after delayed diagnosis of tuberculosis. Infection 2002; 30: 46-49.

25. Ledru E, Ledru S, Zoubga A: Granuloma formation and tuberculosis transmission in HIV-infected patients. Immunol Today 1999; 20: 336-337.

26. De Vuyst D, Vanhoenacker F, Gielen J, Bernaerts A, De Schepper AM: Imaging features of musculoskeletal tuberculosis. Eur Radiol 2003; 13: 1809-1819.

27. Tehranzadeh J, Ter-Oganesyan RR, Steinbach LS: Musculoskeletal disorders associated with HIV infection and AIDS. Part I: Infectious musculoskeletal conditions. Skelet Rad 2004; 33: 249-259.

28. Spinner RJ, Sexton DJ, Goldner RD, Levin LS: Periprosthetic infections due to Mycobacterium tuberculosis in patients with no prior history of tuberculosis. J Arthroplasty 1996; 11: 217-222. 